\title{
THE COSET LATTICES OF E. S. BARNES AND G. E. WALL
}

\author{
ALEXANDER J. HAHN
}

(Received 19 June 1989; revised 10 October 1989)

\author{
Communicated by R. Lidl \\ Dedicated to G. E. (Tim) Wall, in recognition of his distinguished contribution to \\ mathematics in Australia, on the occasion of his retirement
}

\begin{abstract}
John Conway's analysis in 1968 of the automorphism group of the Leech lattice and his discovery of three sporadic simple groups led to the immediate speculation that other $\mathbb{Z}$-lattices might have interesting automorphism groups which give rise to (possibly new) finite simple groups. (The classification theorem for the finite simple groups has since told us that no new finite simple groups can arise in this or any other way.) For example in 1973, M. Broue and $M$. Enguehard constructed, in every dimension $2^{n}$, an even lattice (unimodular if $n$ is odd) whose automorphism group is related to the simple Chevalley group of type $D_{n}$. This family of integral lattices received attention and acclaim in the subsequent literature. What escaped the attention of this literature, however, was the fact that these lattices had been discovered years earlier. Indeed in 1959, E. S. Barnes and G. E. Wall gave a uniform construction for a large class of positive definite $\mathbb{Z}$-lattices in dimensions $2^{n}$ which include those of Broué and Enguehard as special cases. The present article introduces an abstracted and generalized version of the construction of Barnes and Wall. In addition, there are some new observations about BarnesWall lattices. In particular, it is shown how to associate to each such lattice a continuous, piecewise linear graph in the plane from which all the important properties of the lattice, for example, its minimum, whether it is integral, unimodular, even, or perfect can be read off directly.
\end{abstract}

1980 Mathematics subject classification (Amer. Math. Soc.) (1985 Revision): 11 E 12, 11 E 25,

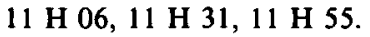

I wish to add a personal salute to Tim Wall on the occasion of his retirement. With the very elevated level of his research and with the distinguished quality of his character he has made a profound and lasting contribution to Australian mathematics.

(C) 1990 Australian Mathematical Society 0263-6115/90 \$A2.00+0.00 
It was already pointed out that the substance of the work of Broue and Enguehard [2] is contained in Barnes and Wall [1, 13]. Figures 1 and 2 below illustrate how the lattices of Broue and Enguehard fit into the bigger picture considered by Barnes and Wall. However, [2] is completely independent and also establishes the connections between Barnes-Wall lattices and Reed-Muller codes. Refer to [4] and its vast bibliography for the extensive and interrelated theories of integral lattices, error correcting codes, and sphere packings. The references of this article add a few entries to this bibliography. I thank the referee for pointing out the articles of Forney [5] and [6]. These papers are of particular interest in the present context since they are based to significant degree on the work of Barnes and Wall already referred to. Incidentally, the "coset construction" in [5] and [6] is completely unrelated to that of the present article. The terminology, notation and basic facts used below will follow O'Meara [10], in particular Part IV. See also Milnor and Husemoller [8].

\section{Basic concepts and coset lattices}

Let $V$ be a $k$ (finite) dimensional vector space over $\mathbb{Q}$ and let

$$
b: V \times V \rightarrow \mathbb{Q}
$$

be a symmetric bilinear form on $V$. We assume throughout that $b$ is positive definite, that is, that the completion $(\bar{V}, \bar{b})$ of $(V, b)$ to $\mathbb{R}$ satisfies $\bar{b}(x, x)>0$ for all $x$ in $\bar{V}$ with $x \neq 0$. For any basis $X=\left\{x_{1}, \ldots, x_{k}\right\}$ of $V$ and any positive $r$ in $\mathbb{Q}$, a positive definite symmetric bilinear form $b$ on $V$ is defined by setting $b\left(x_{i}, x_{j}\right)=0$ for $i \neq j$ and $b\left(x_{i}, x_{i}\right)=r$. The notation

$$
V \cong\langle r\rangle \perp \cdots \perp\langle r\rangle \text { in } X
$$

means that $b$ has been constructed in this way. Let $L$ be a $\mathbb{Z}$-lattice in $V$. So $L$ is a finitely generated $\mathbb{Z}$-module contained in $V$ with operations induced from $V$. Since $L$ is torsion free, $L$ is free. If $L$ spans $V$ over $\mathbb{Q}$ we say that $L$ is on $V$. In this case, $\operatorname{rank}_{\mathrm{z}} L=\operatorname{dim}_{\mathbb{Q}} V=k$. The set

$$
L^{*}=\{y \in V \mid b(x, y) \in \mathbb{Z} \text { for all } x \text { in } L\}
$$

is also a $\mathbb{Z}$-lattice in $V$. It is called the dual lattice of $L$. The lattice $L$ is called integral if $b(x, y) \in \mathbb{Z}$ for all $x$ and $y$ in $L$, that is if $L \subseteq L^{*}$, and $L$ is called unimodular (or non-singular) if $L=L^{*}$. If $L$ is integral, then $L$ is called even if $b(x, x) \in 2 \mathbb{Z}$ for all $x$ in $L$, and odd otherwise. Assume now that $L$ is on $V$. Let $X=\left\{x_{1}, \ldots, x_{k}\right\}$ span $L$ over $\mathbb{Z}$. Since it spans $V$ over $\mathbb{Q}, X$ is a basis of $L$. The $k \times k$ matrix $A=\left(b\left(x_{i}, x_{j}\right)\right)$ is called 
the matrix of $L$ in the base $X$. We write $L \sim A$ in $X$. Observe that $L$ is integral if and only if all entries of $A$ are in $\mathbb{Z}$. The element $\operatorname{det} A \in \mathbb{Q}$ is independent of the choice of $X$ and we define $\operatorname{det} L=\operatorname{det} A$. One finds that $\operatorname{det} L^{\#}=(\operatorname{det} L)^{-1}$. If $L$ is integral, then $\operatorname{det} L$ is a positive integer and

$$
\left[L^{\#}: L\right]=\operatorname{det} L \text {. }
$$

An integral lattice $L$ is called $p$-elementary if the Abelian group $L^{\#} / L$ is an elementary $p$-group. If $N$ and $M$ are submodules of $L$ such that $L=$ $N \oplus M$ and $b(x, y)=0$ for all $x$ in $N$ and $y$ in $M$, we write $L=N \perp M$ and refer to this as a splitting of $L$. If $L$ has such a splitting with both $N$ and $M$ non-zero, $L$ is called decomposable; $L$ is called indecomposable if it is not decomposable. A theorem of Eichler asserts that any $L$ has a splitting $L=L_{1} \perp \cdots \perp L_{m}$ into indecomposable lattices in $V$ which is unique up to the order of the components. The smallest value in $\{b(x, x) \mid x \neq 0$ in $L\}$ is denoted $\min L$. We will call a vector $x \in L$ a minimal vector if $b(x, x)=\min L$. The lattice $L$ is called perfect, if $h: V \times V \rightarrow \mathbb{Q}$ given by $h(x, y)=0$ for all $x$ and $y$, is the only symmetric bilinear form on $V$ such that $h(x, x)=0$ for all the minimal vectors $x$ of $L$. It is not hard to see that if $L$ is perfect, then $L$ is indecomposable.

A general method of constructing positive definite $\mathbb{Z}$-lattices follows. Let $G$ be a finite set of $k$ elements. Let $e$ be a distinguished element of $G$ and fix an ordering $G=\left\{e=g_{0}, g_{1}, \ldots, g_{k-1}\right\}$ of $G$. For the moment $G$ and $e$ will be arbitrary, but soon $G$ will be taken to be a multiplicative group and $e$ its identity element. Let $\mathbf{C}$ be a collection of non-empty subsets of $G$ containing $\{e\}$. Let $\mathbb{N}$ be the set of positive integers and let $\lambda: \mathbf{C} \rightarrow \mathbb{N}$ be any function that satisfies:

(1) $\operatorname{card} C \leq \operatorname{card} D \Rightarrow \lambda(D) \leq \lambda(C)$,

(2) $\operatorname{card} C|\operatorname{card} D \Rightarrow \lambda(D)| \lambda(C)$.

Note that $\operatorname{card} C=\operatorname{card} D$ implies that $\lambda(C)=\lambda(D)$, and that $\lambda(C) \mid \lambda(e)$ for all $C$ in $\mathbf{C}$. Denoting the set of (positive) divisors of $\lambda(e)$ by $\operatorname{div} \lambda(e)$ we see that $\lambda: \mathbf{C} \rightarrow \operatorname{div} \lambda(e)$. Let $V$ be a vector space over $\mathbb{Q}$ with basis $\left\{e=g_{0}, g_{1}, \ldots, g_{k-1}\right\}$ and define a positive definite $b$ on $V$ by

$$
V \cong\left\langle\frac{1}{\lambda(e)}\right\rangle \perp \cdots \perp\left\langle\frac{1}{\lambda(e)}\right\rangle
$$

in the basis $\left\{g_{0}, \ldots, g_{k-1}\right\}$. When emphasizing the role of $\lambda$ we will denote the form $b$ by $b_{\lambda}$ and $V$ by $V_{\lambda}$. For any non-empty subset $S$ of $G$ denote by $\mathbf{v}_{S}$ the vector

$$
\mathbf{v}_{S}=\sum_{g \in S} g
$$


in $V$. Let $L(G, \lambda)$ be the $\mathbb{Z}$-lattice in $V$ spanned by $\left\{\lambda(C) \mathbf{v}_{C} \mid C \in \mathbf{C}\right\}$.

EXAMPLE 1. The terminology, notation and theorems cited in this example come from [4, Chapter 10]. Let $G=\{e=\infty, 0,1,2, \ldots, 22\}$ be the projective line over the field $\mathbb{F}_{23}$. Let $\mathbf{C}$ consist of $\{e\}$ and the special octads. Define $\lambda$ on $\mathbf{C}$ by $\lambda(e)=8$ and $\lambda(C)=2$ for any special octad $C$. Let $\mathbf{C}^{\prime}$ consist of $\mathbf{C}$ and $G$ and extend $\lambda$ to a map $\lambda^{\prime}$ on $\mathbf{C}^{\prime}$ by setting $\lambda^{\prime}(G)=1$. Note that $L\left(G, \lambda^{\prime}\right)=\mathbb{Z}_{G}+L(G, \lambda)$. The lattices $L(G, \lambda)$ and $L\left(G, \lambda^{\prime}\right)$ are closely related to the Leech lattice. The connection is as follows: let $\Lambda_{0}$ be the sublattice of $L(G, \lambda)$ spanned by all the $\lambda(C) V_{C}$ with $C \neq\{e\}$. This is the lattice $\Lambda_{0}$ (scaled by $\frac{1}{8}$ ) of [4, Chapter 10]. Clearly,

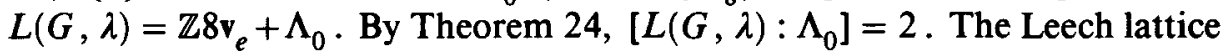
is the lattice $\Lambda=\mathbb{Z}\left(\mathbf{v}_{G}-4 v_{e}\right)+\Lambda_{0}$. It is unimodular. By Theorem $25, L(G, \lambda)$ is contained in $\Lambda$. So $L(G, \lambda)$ is integral and it follows that $L\left(G, \lambda^{\prime}\right)$ is integral also. By Theorem 24, $\left[\Lambda: \Lambda_{0}\right]=4$, and therefore $[\Lambda: L(G, \lambda)]=2$. Again by Theorem $24,2 \mathbf{v}_{G} \in \Lambda_{0}$. So $\left[L\left(G, \lambda^{\prime}\right): L(G, \lambda)\right]$ is 1 or 2 . By Theorem $25, \mathbf{v}_{G} \notin \Lambda$, so this index must be 2 . Note that $L(G, \lambda)^{\#}$ contains $L\left(G, \lambda^{\prime}\right)$ and $\Lambda$. Since $\Lambda$ is unimodular and $\left[\Lambda: \Lambda_{0}\right]=4,\left[\left(\Lambda_{0}\right)^{\#}: \Lambda_{0}\right]=$ 16. In brief, we have

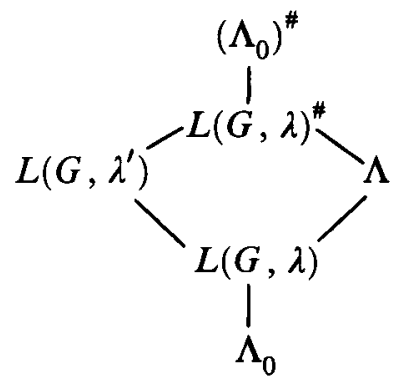

where all the indicated indices are equal to 2 . Note that $L\left(G, \lambda^{\prime}\right)$ is unimodular (and odd), and that $L\left(G, \lambda^{\prime}\right)$ and $\Lambda$ are neighbor lattices. See [9], particularly Section 8 of II.

From now on $G$ will be a (multiplicative) group of finite order $k$ and $e$ will be its identity element. Let $\mathbf{C}$ be the set of all the cosets (relative to all the subgroups) of $G$ and

$$
\lambda: \mathbf{C} \rightarrow \mathbb{N}
$$

any function that satisfies (1) and (2) above. By Lagrange's theorem and (2), $\lambda(G) \mid \lambda(C)$ for all $C$ in $C$. Without essential loss of generality, we therefore normalize $\lambda$ and assume that $\lambda(G)=1$. Let $\mathbf{G} \subseteq \mathbf{C}$ be the set of subgroups of $G$. Note that in order to define a function $\lambda$ on $\mathbf{C}$, it suffices to define $\lambda$ on $\mathbf{G}$. Since it contains the vectors $\lambda\left(g_{i}\right) g_{i}=\lambda(e) g_{i}$ for $1 \leq i \leq k$, the lattice $L(G, \lambda)$ is on $V_{\lambda}$. 
Lemma 1.1. Let $g H$ and $g^{\prime} K$, with $g$ and $g^{\prime}$ in $G$ and $H$ and $K$ in $\mathbf{G}$, be elements in C. Assume that $g H \cap g^{\prime} K \neq \varnothing$, and put $g h=g^{\prime} k$ for some $h$ in $H$ and $k$ in $K$. Then $g H \cap g^{\prime} K=g h(H \cap K)$.

Proof. Since $g^{\prime}=g h k^{-1}, g H \cap g^{\prime} K=g H \cap g h K$. If $g h^{\prime}=g h k^{\prime}$ is an arbitrary element in this intersection, then $h^{\prime}=h k^{\prime}$, so $k^{\prime} \in H \cap K$. So $g h^{\prime}=g h k^{\prime}$ is in $g h(H \cap K)$. This provides one inclusion. The other is trivial.

Proposition 1.2. $L(G, \lambda)^{\#}=\left\{x=\sum_{g \in G} a_{g} g \in V \mid \lambda(C) \sum_{g \in C} a_{g} \in \lambda(e) \mathbb{Z}\right.$ for all $C$ in $\mathbf{C}$.

Proof. Let $x \in V$ and put $x=\sum_{g \in G} a_{g} g$. Then $x \in L(G, \lambda)^{\#}$ if and only if $b\left(\sum_{g \in G} a_{g} g, \lambda(C) \mathbf{v}_{C}\right) \in \mathbb{Z}$ for all $C$ in $\mathbf{C}$. Since

$$
\begin{aligned}
b\left(\sum_{g \in G} a_{g} g, \lambda(C) \mathbf{v}_{C}\right) & =b\left(\sum_{g \in C} a_{g} g, \lambda(C) \mathbf{v}_{C}\right) \\
& =\lambda(C) \sum_{g \in C} a_{g} b\left(g, \mathbf{v}_{C}\right)=\frac{\lambda(C)}{\lambda(e)} \sum_{g \in C} a_{g},
\end{aligned}
$$

$x \in L(G, \lambda)^{\#}$ if and only if $\lambda(C) \sum_{g \in C} a_{g} \in \lambda(e) \mathbb{Z}$ for all $C$ in $\mathbf{C}$.

Proposition 1.3. The lattice $L(G, \lambda)$ is integral if and only if

$$
\lambda(e) \mid \lambda(H) \lambda(K) \operatorname{card}(H \cap K)
$$

for all $H$ and $K$ in $\mathbf{G}$.

Proof. For $C$ and $D$ in $\mathbf{C}$ arbitrary,

$$
b\left(\lambda(C) \mathbf{v}_{C}, \lambda(D) \mathbf{v}_{D}\right)=\lambda(C) \lambda(D) \operatorname{card}(C \cap D) \frac{1}{\lambda(e)} .
$$

So $V$ is integral if and only if this element is in $\mathbb{Z}$ for any $C$ and $D$ in C. It remains to prove that if this element is in $\mathbb{Z}$ for any $H$ and $K$ in $\mathbf{G}$, then this is so for any $C$ and $D$ in $C$. But this is an easy consequence of Lemma 1.1 above.

Corollary 1.4. If $L(G, \lambda)$ is integral, then $\lambda(e) \mid \lambda(C)$ card $C$ for all $C$ in C. In particular, $\lambda(e) \mid \operatorname{card} G$.

Assume that $G$ is Abelian. So for each divisor of $m$ of card $G$ there is a subgroup $H$ of $G$ of order $m$. It follows that for any subgroup $H$ of 
$G$ there is a subgroup $H^{\prime}$ of $G$, such that $\operatorname{card} H \cdot \operatorname{card} H^{\prime}=\operatorname{card} G$. Set $\lambda^{\prime}(H)=\lambda(e) / \lambda\left(H^{\prime}\right)$. Note that $\lambda^{\prime}(H)$ is independent of the particular $H^{\prime}$ chosen. We have defined $\lambda^{\prime}: \mathbf{C} \rightarrow \mathbb{N}$. Clearly, $\lambda^{\prime}(e)=\lambda(e), \lambda^{\prime}(G)=1$, and $\lambda^{\prime}$ satisfies defining property (1). It also satisfies (2). To check this, it suffices to consider $C$ and $D$ in $\mathbf{G}$ and to assume that $C \subseteq D$. Put card $D=m$ card $C$. Note that card $C^{\prime}=m$ card $D^{\prime}$. So $\lambda\left(C^{\prime}\right) \mid \lambda\left(D^{\prime}\right)$, and hence $\lambda^{\prime}(D) \mid \lambda^{\prime}(C)$. Since $\lambda^{\prime}(e)=\lambda(e), L\left(G, \lambda^{\prime}\right)$ is also a lattice on the quadratic space $V_{\lambda}$.

EXAmple 2. Suppose $G=G_{p}$ is the cyclic group of prime order $p$. So card $G=k=p$. Set $\lambda(G)=1$ and $\lambda(e)=p$. By Corollary $1.4, \lambda(e)=p$ is the only choice if $L(G, \lambda)$ is to be integral and non-trivial. It is clear that $L(G, \lambda)$ is spanned by $X=\left\{\mathbf{v}_{G}, p g_{1}, \ldots, p g_{k-1}\right\}$. Hence $X$ is a basis of $L(G, \lambda)$. Easy computations show that in the basis $Y=\left\{\mathbf{v}_{G}, p g_{1}-\right.$ $\left.\mathbf{v}_{G}, \ldots, p g_{k-1}-\mathbf{v}_{G}\right\}, L(G, \lambda) \sim[1] \perp A$, where $A$ is the $(p-1) \times(p-1)$ matrix

$$
\left[\begin{array}{cccc}
p-1 & -1 & \ldots & -1 \\
-1 & p-1 & \ldots & -1 \\
& & \ddots & \\
-1 & -1 & & p-1
\end{array}\right] .
$$

Row and column reducing $A$ appropriately shows that $\operatorname{det} L(G, \lambda)=p^{(p-2)}$.

Example 3. Let $G=G_{p} \times G_{p}$ be the product of two cyclic groups of prime order $p$. So $k=p^{2}$. Set $\lambda(G)=1, \lambda(e)=p^{2}$ and $\lambda(H)=p$ for any subgroup $H$ of order $p$. Since $G$ is a 2-dimensional vector space over the field $\mathbb{F}_{p}$, there are exactly $\left(p^{2}-1\right) /(p-1)=p+1$ subgroups of order $p$. Fix a subgroup $H$ of order $p$ and observe that there are $p-1$ cosets of the form $g H$ with $g H \neq H$. As $H$ varies over the $p+1$ subgroups of order $p$, we get $(p+1)(p-1)=p^{2}-1$ such cosets by Lemma 1.1. Denote them by $C_{1}, C_{2}, \ldots, C_{k-1}$. Put $X=\left\{\mathbf{v}_{G}, p \mathbf{v}_{C_{1}}, \ldots, p \mathbf{v}_{C_{k-1}}\right\}$. We claim that $X$ spans $L(G, \lambda)$. It is clear that $p v_{H}$ is in the span of $X$ for any subgroup $H$ of order $p$. Now fix $g$ in $G$. There are exactly $p+1$ cosets (including subgroups) of order $p$ containing $g$; their union is $G$. This follows by translation from the case $g=e$. Denote them by $C_{1}, \ldots, C_{p+1}$. By Lemma 1.1, these $p$ cosets intersect pairwise in the element $g$ only. It follows that

$$
\sum_{i=1}^{p+1} p \mathbf{v}_{C_{i}}=(p+1) p g+\sum_{h \neq g} p h=p^{2} g+p \mathbf{v}_{G} .
$$

So $p^{2} g=\lambda(g) g$ is in the span of $X$. So $X$ spans $L(G, \lambda)$ and is therefore 
a basis of $L(G, \lambda)$. Clearly, $Y=\left\{\mathbf{v}_{G}, p \mathbf{v}_{C_{1}}-\mathbf{v}_{G}, \ldots, p \mathbf{v}_{C_{k-1}}-\mathbf{v}_{G}\right\}$ is also a basis of $L(G, \lambda)$. Let $H$ and $K$ be distinct subgroups of order $p$ and let $C=g H$ and $D=g^{\prime} K$ be arbitrary cosets. Since $G$ is Abelian and $G=H K$, it follows easily that $C \cap D \neq \varnothing$. So by Lemma 1.1, $C \cap D$ is a point. Let $H_{1}, \ldots, H_{p+1}$ be the distinct subgroups of order $p$, and reorder the non-trivial cosets $C_{1}, C_{2}, \ldots, C_{k-1}$ in such a way that $C_{1}, \ldots, C_{p-1}$ belong to $H_{1}$, and $C_{p}, \ldots, C_{2 p-2}$, belong to $H_{2}$, and so on. Reorder the elements in $Y$ accordingly. A routine computation shows that in this basis,

$$
L(G, \lambda) \sim[1] \perp A \perp \cdots \perp A,
$$

where $A$ is the $(p-1) \times(p-1)$ matrix of Example 2, repeated $p+1$ times in the decomposition. Observe that $L(G, \lambda)$ is integral. Since $\operatorname{det} A=p^{p-2}$, $\operatorname{det} L(G, \lambda)=p^{(p-2)(p+1)}$.

Are the lattices in Examples 2 and 3 -elementary? Does the pattern exhibited in Examples 2 and 3 continue for $p$-groups of larger orders? What happens for other choices of $\lambda$ ? Is an integral $L(G, \lambda)$-elementary whenever $G$ is an elementary $p$-group?

\section{The lattices of Barnes and Wall}

We now turn to the lattices of Barnes and Wall. This is the situation where the group $G$ is taken to be an elementary Abelian 2-group. Note that any elementary Abelian 2-group is a vector space over $\mathbb{F}_{2}$ in a natural way, and conversely. The subspaces are the subgroups.

Definition. The lattice $L(G, \lambda)$ is a Barnes-Wall lattice if

(i) $G$ is a (finite) elementary Abelian 2-group,

(ii) $\lambda(e)$ is a power of 2 , and

(iii) $\lambda: \mathbf{C} \rightarrow \operatorname{div} \lambda(e)$ is onto.

By Corollary 1.4, an integral $L(G, \lambda)$ is a Barnes-Wall lattice if and only if (i) and (iii) hold. Note that $\lambda(G)=1$ follows from (iii) and does not have to be assumed.

For the rest of the paragraph we fix an elementary Abelian 2-group $G$ of order $k=2^{n}$. Any coset in $G$ has cardinality $2^{r}, 0 \leq r \leq n$. The typical such coset will be denoted by $C_{r}$. We begin by showing that the definition above is equivalent to that of [1]. Suppose that $\lambda$ with the indicated properties is given. Set $\lambda\left(C_{r}\right)=2^{\lambda_{n-r}}$. Note that $1=\lambda(G)=2^{\lambda_{0}}$ and that $\lambda(e)=2^{\lambda_{n}}$. In view of the defining properties (1) and (2),

$$
0=\lambda_{0} \leq \lambda_{1} \leq \cdots \leq \lambda_{i} \leq \cdots \leq \lambda_{n}
$$


is the complete set of images of $\lambda$. Since $\lambda$ is onto, it follows that $\lambda_{i}-\lambda_{i-1} \leq$ 1. The exponents $\lambda_{i}$ therefore satisfy conditions (3.1) of [1]. Note that $\lambda_{n} \leq n$. Conversely, given any such sequence, and defining $\lambda\left(C_{r}\right)=2^{\lambda_{n-r}}$ and, in particular $\lambda(e)=\lambda\left(C_{0}\right)=2^{\lambda_{n}}$, provides a surjective function $\lambda: \mathbf{C} \rightarrow$ $\operatorname{div} \lambda(e)$. It remains to note that the lattice $\Lambda(\lambda)$ of [1, Section 3$]$ is precisely the lattice obtained by scaling $L(G, \lambda)$ by $\lambda(e)$. Observe that the lattice $L\left(G, \lambda^{\prime}\right)$ of Section 1 is also a Barnes-Wall lattice. The associated scalars are $\lambda_{i}^{\prime}=\lambda_{n}-\lambda_{n-i}$. This is the scaled version of the lattice $\Lambda\left(\lambda^{\prime}\right)$ of [1].

We now describe some of the important results of [1]. Note that the Barnes-Wall lattices that are considered here differ from the original versions in that we have scaled by $\frac{1}{\lambda(e)}=2^{-\lambda_{n}}$. The formulation of the results below differs accordingly.

Let $L(G, \lambda)$ be a Barnes-Wall lattice. Since the 2-group $G$ is determined uniquely (up to isomorphism) by its order, we denote $L(G, \lambda)$ by $L_{k}(\lambda)$. Of course, $\operatorname{rank}_{\mathrm{z}} L_{k}(\lambda)=k$. As above, we set $\lambda\left(C_{r}\right)=2^{\lambda_{n-r}}$. So $\lambda(e)=$ $\lambda\left(C_{0}\right)=2^{\lambda_{n}}$. It is clear that there are elements $\left\{x_{1}, \ldots, x_{n}\right\}$ in $G$ such that every $g$ in $G$ is uniquely of the form

$$
g=\left(x_{1}\right)^{\varepsilon_{1}}\left(x_{2}\right)^{\varepsilon_{2}} \cdots\left(x_{n}\right)^{\varepsilon_{n}},
$$

where $\varepsilon_{i}$ is either 0 or 1 . In vector space terminology $X=\left\{x_{1}, \ldots, x_{n}\right\}$ is a basis of $G$ over $\mathbb{F}_{2}$. Including the empty set, there are exactly $2^{n}$ subsets of $X$. Let $\mathbf{H}$ be the collection (there are of course $2^{n}$ ) of subgroups of $G$ that are generated by these $2^{n}$ subsets. If $H$ in $\mathbf{H}$ has order $2^{r}$, we denote $H$ by $H_{r}$.

THEOREM 2.1. (1) $L_{k}\left(\lambda^{\prime}\right)=L_{k}(\lambda)^{\#}$,

(2) $B=\left\{2^{\lambda_{n-r}} \mathbf{v}_{H_{r}} \mid H_{r} \in \mathbf{H}\right\}$ is a basis of $L_{k}(\lambda)$, and

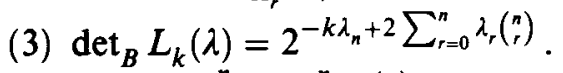

Note that $k=2^{n}=\sum_{r=0}^{n}\left(\begin{array}{l}n \\ r\end{array}\right)$.

Proof. This is [1, Theorem 3.1]. We sketch the proof. It is easy to see that $L_{k}\left(\lambda^{\prime}\right) \subseteq L_{k}(\lambda)^{\#}$. Let $\Gamma$ be the $\mathbb{Z}$-lattice spanned by the basis $\left\{e=g_{0}, g_{1}, \ldots, g_{k-1}\right\}$ of $V_{\lambda}$. Note that $\operatorname{det} \Gamma=\left(2^{-\lambda_{n}}\right)^{k}=2^{-k \lambda_{n}}$. It is not very hard to show that $\left\{\mathbf{v}_{H_{r}} \mid H_{r} \in \mathbf{H}\right\}$ is a basis of $\Gamma$. So the determinant of the matrix of the form $b_{\lambda}$ in this basis is $2^{-k \lambda_{n}}$ also. Now arrange the vectors in $B$ so that the indices $r$ occur in non-decreasing order and denote by $N$ the $\mathbb{Z}$-lattice spanned by the vectors in $B$. Note that $N \subseteq L_{k}(\lambda) \subseteq \Gamma$. Using [10, $\S 82 \mathrm{E}]$, one checks that the volume of $N$ is the ideal of $\mathbb{Z}$ generated by

$$
2^{-k \lambda_{n}+2 \sum_{r=0}^{n} \lambda_{n-r}\left(\begin{array}{l}
n \\
r
\end{array}\right)}=2^{-k \lambda_{n}+2 \sum_{s=0}^{n} \lambda_{s}\left(\begin{array}{c}
n \\
n-s
\end{array}\right)}=2^{-k \lambda_{n}+2 \sum_{r=0}^{n} \lambda_{r}\left(\begin{array}{l}
n \\
r
\end{array}\right)} .
$$


Let $B^{\prime}$ be the set of vectors obtained by replacing $\lambda_{n-r}$ by $\lambda_{n-r}^{\prime}$ and let $N^{\prime} \subseteq L_{k}\left(\lambda^{\prime}\right)$ be the analogue of $N$. The volume of $N^{\prime}$ has an expression analogous to that of $N$ and a routine computation shows that the product of these volumes is $\mathbb{Z}$. By $[10, \S 82 \mathrm{~F}], N^{\#}$ and $N^{\prime}$ have the same volume. Since $N^{\prime} \subseteq L_{k}\left(\lambda^{\prime}\right) \subseteq L_{k}(\lambda)^{*} \subseteq N^{*}, N^{\prime} \subseteq N^{*}$. So by [10, $\left.\S 82: 11 \mathrm{a}\right], N^{*}=N^{\prime}$ and hence $N^{\prime}=L_{k}\left(\lambda^{\prime}\right)=L_{k}(\lambda)^{\#}=N^{\#}$. So $L_{k}(\lambda)=N$. This proves (1) and (2). Computing the volume of $L_{k}(\lambda)$ in the basis $B$ provides (3).

Corollary 2.1A.

$$
L_{k}(\lambda)=\left\{x=\sum_{g \in G} a_{g} g \in V \mid \sum_{g \in C_{r}} a_{g} \in 2^{\lambda_{r}} \mathbb{Z} \text { for all } C_{r} \text { in } \mathbf{C}\right\} .
$$

Proof. By 2.1, $L_{k}(\lambda)=L_{k}\left(\lambda^{\prime}\right)^{\#}$. Now apply Proposition 1.2.

Corullary 2.1B. Let $C_{r}$ be a coset in $G$. Then $2^{i} \mathbf{v}_{C_{r}} \in L_{k}(\lambda)$ if and only if $i \geq \lambda_{n-r}$.

Proof. Since $\lambda\left(C_{r}\right)=2^{\lambda_{n-r}}, 2^{i} \mathbf{v}_{C_{r}} \in L_{k}(\lambda)$ if $i \geq \lambda_{n-r}$. Now let $2^{i} \mathbf{v}_{C_{r}} \in$ $L_{k}(\lambda)$. Let $D=D_{s}$ be any coset contained in $C=C_{r}$. Put $C=g H$ and $D=g^{\prime} K$ with $K$ and $H$ in G. Since $g^{\prime} \in C, C=g^{\prime} H$. So $K$ is a subgroup of $H$. Let $N$ be a complement of $H$ in $G$ and consider the coset $E=E_{t}=g^{\prime} K N$. Note $t=s+n-r$. By Lemma 1.1, $C \cap E=g^{\prime} H \cap g^{\prime} K N=$ $g^{\prime}(H \cap K N)=D$. So $2^{s} 2^{i} \in 2^{\lambda_{s+n-r}} \mathbb{Z}$ by Corollary $2.1 \mathrm{~A}$. This is so for any $s$ with $0 \leq s \leq r$. Taking $s=0$, shows that $i \geq \lambda_{n-r}$.

Consider the sequence $0=\lambda_{0} \leq \lambda_{1} \leq \cdots \leq \lambda_{i} \leq \cdots \leq \lambda_{n}$ given by $\lambda$. For any $i, 0 \leq i \leq n$, set $\bar{\lambda}_{i}=i-\lambda_{i}$. Let $i<j$. Since $\lambda_{j}-\lambda_{i} \leq j-i$, $\bar{\lambda}_{i}=i-\lambda_{i} \leq j-\lambda_{j}=\bar{\lambda}_{j}$. Since $\lambda_{i+1}-\lambda_{i} \leq 1, \bar{\lambda}_{i+1}-\bar{\lambda}_{i}=(i+1)-\lambda_{i+1}-\left(i-\lambda_{i}\right)=$ $1-\left(\lambda_{i+1}-\lambda_{i}\right) \leq 1$. As noted earlier, the sequence $0=\bar{\lambda}_{0} \leq \bar{\lambda}_{1} \leq \cdots \leq \bar{\lambda}_{i} \leq$ $\cdots \leq \bar{\lambda}_{n}=n-\lambda_{n}$ defines a surjective function $\bar{\lambda}: \mathbf{C} \rightarrow \operatorname{div} 2^{n-\lambda_{n}}$ and hence a Barnes-Wall lattice $L_{k}(\bar{\lambda})$.

THEOREM 2.2. The lattices $L_{k}(\lambda)$ and $L_{k}(\bar{\lambda})^{\#}$ are isometric.

The rank of a non-zero vector $x$ in $L_{k}(\lambda)$ is defined to be the largest $i$, $0 \leq i \leq n$, such that all coordinates of $x$ in the basis $\left\{e=g_{0}, g_{1}, \ldots, g_{k-1}\right\}$ of $V$ are divisible by $2^{\lambda_{i}}$. Let $m_{\lambda}$ be given by

$$
m_{\lambda}=\min _{0 \leq i \leq n}\left(2 \lambda_{i}-i\right) \text {. }
$$


THEOREM 2.3. $\min L_{k}(\lambda)=2^{m_{\lambda}+\left(n-\lambda_{n}\right)}$. The minimal vectors of $L_{k}(\lambda)$ of rank $r$ are precisely the vectors in $L_{k}(\lambda)$ of the form $\sum_{g \in S}\left(\varepsilon_{g} 2^{\lambda_{r}}\right) g$, where $0 \leq r \leq n, 2 \lambda_{r}-r=m_{\lambda}, S$ is a subset of $G$ of order $2^{n-r}$, and $\varepsilon_{g}= \pm 1$.

Let $r$, with $0 \leq r \leq n$, satisfy $2 \lambda_{r}-r=m_{\lambda}$. Which of vectors $\sum_{g \in S}\left(\varepsilon_{g} 2^{\lambda_{r}}\right) g$ are actually in $L_{k}(\lambda)$ ? In [13, Section 3.1] it is shown that this is the case if and only if $S$ is a coset and if the function $f: S \rightarrow \mathbb{Z}_{2}$ given by $f(g)=\varepsilon_{g}$ satisfies certain properties. This characterization of the minimal vectors of $L_{k}(\lambda)$ can be used to find a formula for their number. See formula (5.10) of [1].

Suppose $r$ satisfies $0<r<n$ and $2 \lambda_{r}-r=m_{\lambda}$. Consider the set $M_{r}$ of all the vectors of the form $2^{\lambda_{r}} \mathbf{v}_{C_{n-r}}$ and $2^{\lambda_{r}} \mathbf{v}_{C_{n-r-1}}-2^{\lambda_{r}} \mathbf{v}_{D_{n-r-1}}$, where $C_{n-r-1}$ and $D_{n-r-1}$ are distinct cosets belonging to the same subgroup. It is not difficult to see that the set $M_{r}$ is a subset of $L_{k}(\lambda)$. So it consists entirely of minimal vectors of $L_{k}(\lambda)$.

The final result relevant for our purposes asserts that the lattice $L_{k}(\lambda)$ is perfect if such an $r$ exists.

Theorem 2.4. Suppose that $r$ satisfies $0<r<n$ and $2 \lambda_{r}-r=m_{\lambda}$. If $h: V \times V \rightarrow \mathbb{Q}$ is a bilinear form such that $h(x, x)=0$ for all $x$ in $M_{r}$, then $h(x, y)=0$ for all $x$ and $y$ in $V$. In particular, $L_{k}(\lambda)$ is perfect and hence indecomposable.

The lattice $L_{k}(\lambda)$ is in fact extreme whenever the hypothesis of the theorem above holds. Refer to [1] and [13] for this and additional information.

\section{The graph of the lattice $L_{k}(\lambda)$}

Fix $n \geq 1$. A sequence, in present context, is defined to be any ordered $n$-tuple of 0 's and 1's. Let $S=\left[\varepsilon_{1}, \ldots, \varepsilon_{n}\right]$ be a sequence. We denote by $S^{\#}$ the sequence $S^{\#}=\left[\varepsilon_{n}, \ldots, \varepsilon_{1}\right]$, and by $-S$ the sequence $-S=$ $\left[1-\varepsilon_{1}, \ldots, 1-\varepsilon_{n}\right]$.

As in Section 2, we fix an elementary Abelian 2-group $G$ of order $k=2^{n}$ and let $L_{k}(\lambda)=L(G, \lambda)$ be a Barnes-Wall lattice on $V_{\lambda}$. Let $0=\lambda_{0} \leq \lambda_{1} \leq$ $\cdots \leq \lambda_{n}$ be the scalars determined by $\lambda$. The sequence $\operatorname{sq} L_{k}(\lambda)$ of $L_{k}(\lambda)$ is $\left[\varepsilon_{1}, \ldots, \varepsilon_{n}\right]$ defined by

$$
\varepsilon_{1}=\lambda_{1}-\lambda_{0}, \ldots, \varepsilon_{i}=\lambda_{i}-\lambda_{i-1}, \ldots, \varepsilon_{n}=\lambda_{n}-\lambda_{n-1} .
$$


So every Barnes-Wall lattice determines a sequence. Conversely, every sequence $S=\left[\varepsilon_{1}, \ldots, \varepsilon_{n}\right]$ determines a Barnes-Wall lattice $L_{k}(\lambda)$ with $\lambda$ defined by the scalars $\lambda_{i}=\varepsilon_{i}+\cdots+\varepsilon_{i}$.

Proposition 3.1. sq $L_{k}(\lambda)^{\#}=\left(\operatorname{sq} L_{k}(\lambda)\right)^{\#}$.

Proof. By Theorem 2.1, $L_{k}(\lambda)^{\#}=L_{k}\left(\lambda^{\prime}\right)$, where $\lambda_{i}^{\prime}=\lambda_{n}-\lambda_{n-i}$. If $S=\left[\varepsilon_{1}, \ldots, \varepsilon_{n}\right]$ is the sequence of $L_{k}(\lambda)$, then the sequence of $L_{k}\left(\lambda^{\prime}\right)$ is $S^{\prime}=\left[\varepsilon_{1}^{\prime}, \ldots, \varepsilon_{n}^{\prime}\right]$, where $\varepsilon_{i}^{\prime}=\lambda_{i}^{\prime}-\lambda_{i-1}^{\prime}=\left(\lambda_{n}-\lambda_{n-i}\right)-\left(\lambda_{n}-\lambda_{n-i+1}\right)=\varepsilon_{n-i+1}$. So $S^{\prime}=S^{\#}$.

Proposition 3.2. sq $L_{k}(\bar{\lambda})=-\operatorname{sq} L_{k}(\lambda)$.

Proof. Recall, $\bar{\lambda}_{i}=i-\lambda_{i}$. So $\bar{\lambda}_{i}-\bar{\lambda}_{i-1}=i-\lambda_{i}-\left(i-1-\lambda_{i-1}\right)=$ $1-\left(\lambda_{i}-\lambda_{i-1}\right)=1-\varepsilon_{i}$.

Suppose that $L_{k}(\lambda)$ is the lattice that corresponds to $[0, \ldots, 0]$. So all $\lambda_{i}$ are equal to zero. It follows that $\left\{e=g_{0}, g_{1}, \ldots, g_{k-1}\right\}$ is a basis of $L_{k}(\lambda)$ and that in this basis $L_{k}(\lambda) \sim I_{k}$, the $k \times k$ identity matrix. If $L_{k}(\lambda)$ corresponds to $[1, \ldots, 1]$, then by Theorem 2.2 and Propositions 3.1 and 3.2, $L_{k}(\lambda) \sim I_{k}$ in some basis. The lattices corresponding to $[0, \ldots, 0]$ and $[1, \ldots, 1]$ are called the trivial lattices and are denoted $I_{k}$.

Let $S=\left[\varepsilon_{1}, \ldots, \varepsilon_{n}\right]$ be a sequence. For $1 \leq i \leq n$, denote by $p_{i}$ and $q_{i}$ the number of 1 's and 0 's in $\left[\varepsilon_{1}, \ldots, \varepsilon_{i}\right]$. Consider the set of $n+1$ points

$$
(0,0),\left(1, p_{1}-q_{1}\right), \ldots,\left(n, p_{n}-q_{n}\right)
$$

in the $x-y$ plane. The graph $\operatorname{gr} S$, of $S$ is defined to be the continuous, piecewise linear curve obtained by connecting $(0,0)$ to $\left(1, p_{1}-q_{1}\right),\left(1, p_{1}-\right.$ $\left.q_{1}\right)$ to $\left(2, p_{2}-q_{2}\right)$, and so on. Note that $\operatorname{gr} S$ lies in the wedge $0 \leq x \leq n$, $-x \leq y \leq x$. Consider the midpoint $\left(n / 2,\left(p_{n}-q_{n}\right) / 2\right)$ of the segment determined by the points $(0,0)$ and $\left(n, p_{n}-q_{n}\right)$. We define $(\operatorname{gr} S)^{\#}$ to be the graph obtained by reflecting $\operatorname{gr} S$ through this point. It is not difficult to see that $(\operatorname{gr} S)^{\#}=\operatorname{gr}\left(S^{*}\right)$. If $\operatorname{gr} S=(\operatorname{gr} S)^{\#}$, in other words if $\operatorname{gr} S$ is symmetric about the point $\left(n / 2,\left(p_{n}-q_{n}\right) / 2\right)$, then $\left(n / 2,\left(p_{n}-q_{n}\right) / 2\right)$ lies on $\operatorname{gr} S$ and is called the midpoint of $\operatorname{gr} S$. Note that $\operatorname{gr}(-S)=-(\operatorname{gr} S)$, the graph obtained by reflecting $\operatorname{gr} S$ across the line $x=0$. We write $\operatorname{gr} S \leq \operatorname{gr} S^{\prime}$, if the graph of $S$ lies below that of $S^{\prime}$.

The graph of $L_{k}(\lambda)$ is the graph of the sequence of $L_{k}(\lambda)$. We denote it by $\operatorname{gr} L_{k}(\lambda)$. Let $L_{k}(\mu)$ be another Barnes-Wall lattice relative to $G$.

Proposition 3.3. $L_{k}(\lambda) \subseteq L_{k}(\mu)$ if and only if $\operatorname{gr} L_{k}(\lambda) \geq \operatorname{gr} L_{k}(\mu)$. In particular, $L_{k}(\lambda)=L_{k}(\mu)$ if and only if $\operatorname{gr} L_{k}(\lambda)=\operatorname{gr} L_{k}(\mu)$. 
Proof. By use of Corollary 2.1B, $L_{k}(\lambda) \subseteq L_{k}(\mu)$ if and only if $\lambda_{i} \geq \mu_{i}$ for all $i$. This is the same as saying that for all $i$, the $p_{i}$ for $L_{k}(\lambda)$ is greater than or equal to the $p_{i}$ for $L_{k}(\mu)$, or equivalently, that the $q_{i}$ for $L_{k}(\lambda)$ is less than or equal to the $q_{i}$ for $L_{k}(\lambda)$. The rest is clear.

Proposition 3.4. If $\operatorname{gr} L_{k}(\mu)=-\left(\operatorname{gr} L_{k}(\lambda)\right)^{\#}$, then $L_{k}(\mu)$ is isometric to $L_{k}(\lambda)$. So if $L_{k}(\lambda)$ is unimodular and $\operatorname{gr} L_{k}(\mu)=-\operatorname{gr} L_{k}(\lambda)$, then $L_{k}(\mu)$ is isometric to $L_{k}(\lambda)$.

Proof. It suffices to prove the first statement. Using Propositions 3.1 and 3.2 , we get

$$
-\left(\operatorname{gr} L_{k}(\lambda)\right)^{\#}=-\left(\operatorname{gr} L_{k}(\lambda)\right)^{\#}=\left(\operatorname{gr} L_{k}(\bar{\lambda})\right)^{\#}=\operatorname{gr}\left(L_{k}(\bar{\lambda})^{\#}\right) .
$$

So by Proposition 3.3, $L_{k}(\mu)=L_{k}(\bar{\lambda})^{\#}$. Now apply Theorem 2.2.

The following two propositions are easy consequences of Propositions 3.1 and 3.3 and the discussion above.

Proposition 3.5. $L_{k}(\lambda)$ is integral if and only if $\operatorname{gr} L_{k}(\lambda) \geq\left(\operatorname{gr} L_{k}(\lambda)\right)^{\#}$.

Proposition 3.6. $L_{k}(\lambda)$ is unimodular if and only if $\operatorname{gr} L_{k}(\lambda)=\left(\operatorname{gr} L_{k}(\lambda)\right)^{\#}$. So $L_{k}(\lambda)$ is unimodular if and only if the graph of $L_{k}(\lambda)$ is symmetric about the point $\left(n / 2,\left(p_{n}-q_{n}\right) / 2\right)$.

The next two statements are translations of facts from Section 2.

Proposition 3.7. Let $0 \leq i \leq n$. Then $L_{k}(\lambda)$ has minimal vectors of rank $i$ if and only if the graph of $L_{k}(\lambda)$ has an absolute minimum at $x=i$.

Proposition 3.8. If $\operatorname{gr} L_{k}(\lambda)$ has an absolute minimum at $x=i$, with $0<i<n$, then $L_{k}(\lambda)$ is perfect and hence indecomposable.

Recall the parameter $m_{\lambda}$ defined prior to Theorem 2.3. Since $2 \lambda_{i}-i=$ $2 p_{i}-\left(p_{i}+q_{i}\right)=p_{i}-q_{i}$, we find

Proposition 3.9. The minimum $y$-coordinate of the graph of $L_{k}(\lambda)$ is $m_{\lambda}$.

Note that $p_{n}=\lambda_{n}$ is the number of segments of the graph with slope +1 and that this number determines the bilinear form on $V_{\lambda}$. Since $p_{n}+q_{n}=n$, $q_{n}=n-p_{n}=n-\lambda_{n}$. Since $q_{n}$ is the number of 0 's in the sequence of $L_{k}(\lambda), q_{n}$ is the number of segments in the graph of $L_{k}(\lambda)$ with slope -1 . 
Translating Theorem 2.3, we find that

Proposition 3.10. $\min L_{k}(\lambda)=2^{m_{\lambda}+q_{n}}$.

Proposition 3.11. Suppose $L_{k}(\lambda)$ is integral. Then $L_{k}(\lambda)$ is even if and only if $\min L_{k}(\lambda) \geq 2$.

Proof. Since $\left\{2^{\lambda_{n-i}} \mathbf{v}_{C_{i}} \mid C_{i}\right.$ is a subgroup of $\left.G\right\}$ spans $L_{k}(\lambda), L_{k}(\lambda)$ is even if and only if $b\left(2^{\lambda_{n-i}} \mathbf{v}_{C_{i}}, 2^{\lambda_{n-i}} \mathbf{v}_{C_{i}}\right) \in 2 \mathbb{Z}$ for all $i, 0 \leq i \leq n$, and all subgroups $C_{i}$ of $G$. Since

$$
b\left(2^{\lambda_{n-i}} \mathbf{v}_{C_{i}}, 2^{\lambda_{n-i}} \mathbf{v}_{C_{i}}\right)=2^{2 \lambda_{n-i}} b\left(\mathbf{v}_{C_{i}}, \mathbf{v}_{C_{i}}\right)=2^{2 \lambda_{n-i}} 2^{i} 2^{-\lambda_{n}},
$$

$L_{k}(\lambda)$ is even if and only if $2 \lambda_{n-i}+i-\lambda_{n} \geq 1$ for all $i$. But, $2 \lambda_{n-i}+i-\lambda_{n}=$ $2 \lambda_{n-i}-(n-i)-\lambda_{n}+n$. So $L_{k}(\lambda)$ is even if and only if $m_{\lambda}-\lambda_{n}+n \geq 1$, which holds if and only if $m_{\lambda}-p_{n}+p_{n}+q_{n} \geq 1$. Now apply 3.10 .

Proposition 3.12. If $L_{k}(\lambda)$ is non-trivial and unimodular, then $L_{k}(\lambda)$ is even.

Proof. By the proof of the proposition above, $L_{k}(\lambda)$ is even if and only if $2 \lambda_{n-i}+i-\lambda_{n} \geq 1$ for all $i$. Since $L_{k}(\lambda)$ is unimodular, $\lambda_{n-i}=\lambda_{n}-\lambda_{i}$. So $L_{k}(\lambda)$ is even if and only if $\lambda_{n}+i \geq 2 \lambda_{i}+1$ for all $i$. Since it is always the case that $\lambda_{n} \geq \lambda_{i}$ and $i \geq \lambda_{i}, L_{k}(\lambda)$ is even unless $j=\lambda_{j}$ and $\lambda_{n}=\lambda_{j}$ for some $j$. If $j=0$, then $\lambda_{n}=0$. So all $\lambda_{j}$ are 0 , and $\operatorname{sq} L_{k}(\lambda)=[0, \ldots, 0]$. So assume that $j \geq 1$. It follows that $\lambda_{i}=i$ for $i \leq j$, and $\lambda_{i}=\lambda_{n}$ for all $i \geq j$. So $\lambda_{n-i}=0$ for all $i \geq j$. It follows that $j=n$. So sq $L_{k}(\lambda)=[1,1, \ldots, 1]$.

Consider the lattice $L_{k}(\lambda)$ corresponding to $[1,0,0, \ldots, 0,1]$. In view of the propositions above, this lattice is unimodular, even, indecomposable and has minimum 2. Let $V$ be the quadratic space $V \cong\langle 1\rangle \perp \cdots \perp\langle 1\rangle$ in $\left\{e=g_{0}, g_{1}, \ldots, g_{k-1}\right\}$. It follows from the discussion on pages 331 and 332 of [10] in combination with Proposition 1.2, that the isometry $x \rightarrow \frac{1}{2} x$ from the scaled space $V^{1 / 4}$ onto $V$ injects $L_{k}(\lambda)$ into $\Phi_{k}$. Since $L_{k}(\lambda)$ is unimodular and $\Phi_{k}$ indecomposable, it follows that $L_{k}(\lambda)$ is isometric to $\Phi_{k}$. We denote both $L_{k}(\lambda)$ and the isomorphic copy of $L_{k}(\lambda)$ (see Proposition 3.4) corresponding to $[0,1,1, \ldots, 1,0]$ by $\Phi_{k}$.

Figure 1 shows the graphs of the lattices considered by Broue and Enguehard (for $n$ even). It also includes the lattice $\Phi_{k}$ and the two trivial lattices. 


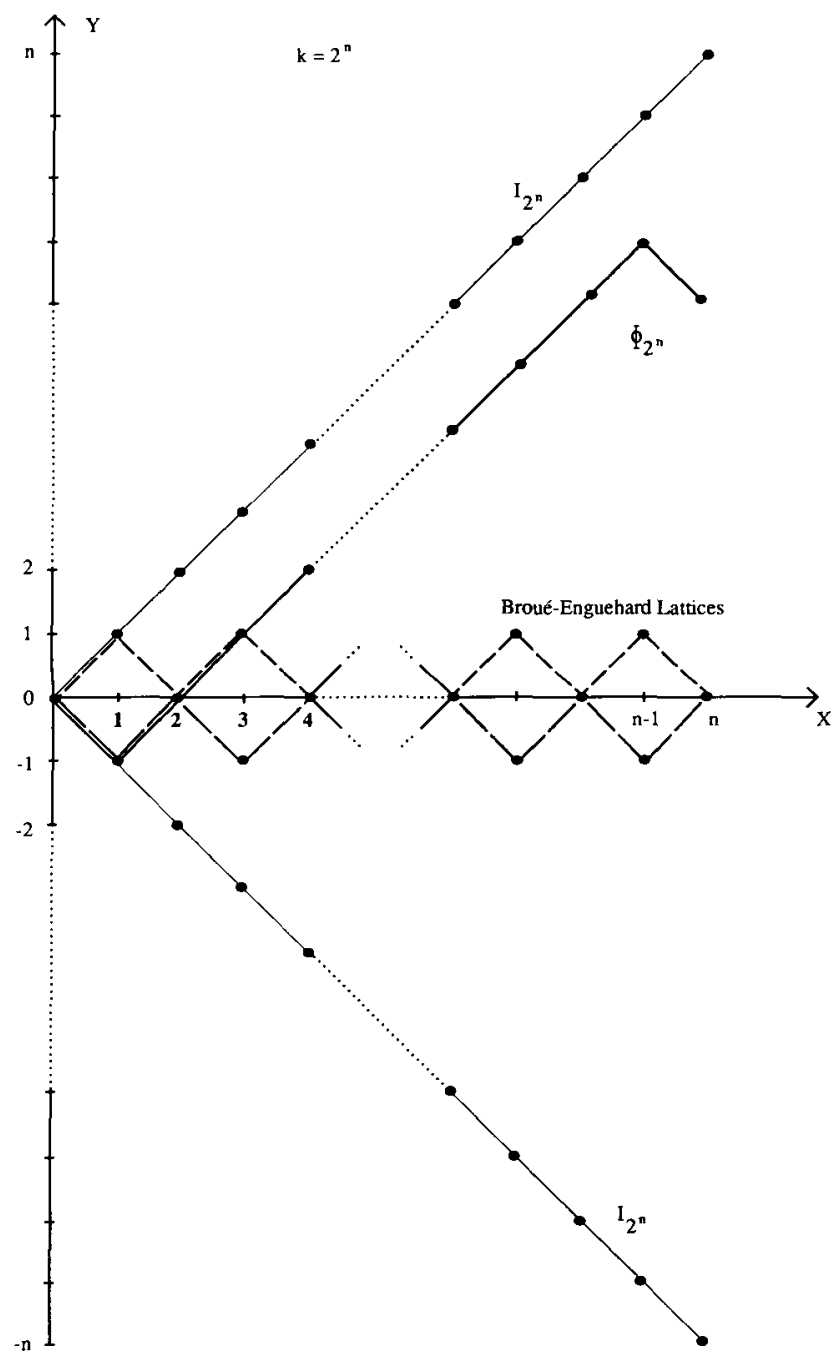

FIGURE 1

Note, in view of 3.6, that the lattice of Broue and Enguehard is unimodular only for odd $n$.

Figure 2 shows the graphs of all (up to the isomorphism provided by Proposition 3.4) the even, unimodular Barnes-Wall lattices in dimension $2^{7}=128$ which have minimal vectors of ranks between 1 and 6 . All these lattices are perfect and hence indecomposable. The lattice (a) is $\Phi_{128}$ and has minimum 2. The lattice (d) is the lattice of Broue and Enguehard and has minimum 8. The lattices (b), (c), and (e) all have minimum 4. Counting the number of 


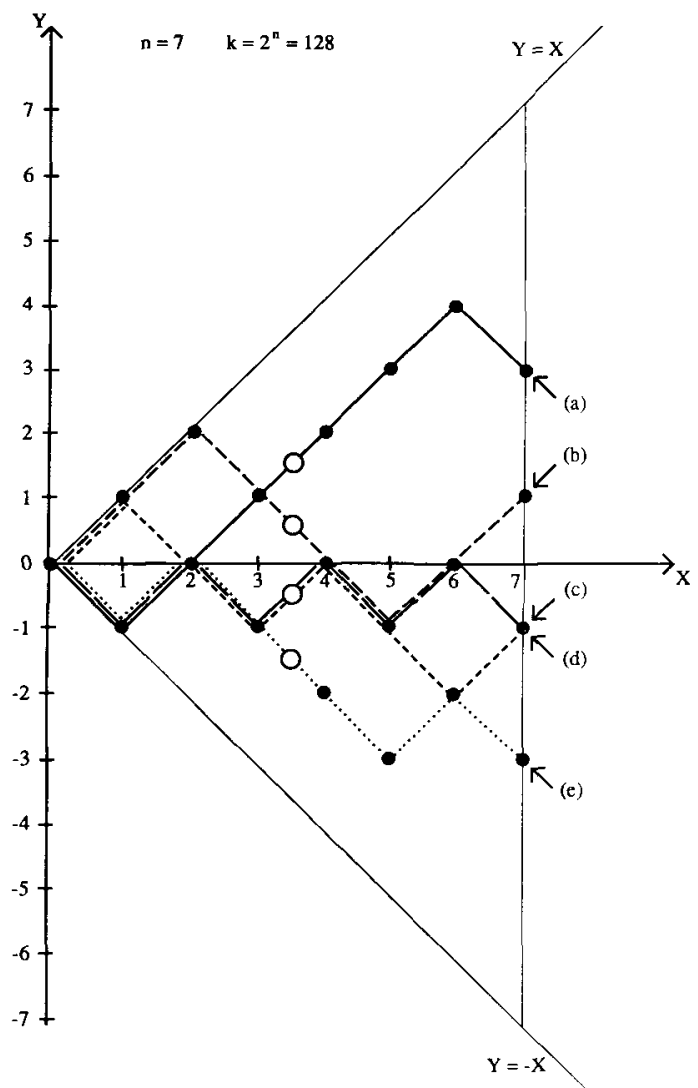

FIGURE 2

minimal vectors (see formula (5.10) of [1]) shows that they are not pairwise isometric. The points $\circ$ are the respective midpoints of the graphs. Their $x$-coordinates are $\frac{7}{2}$.

We conclude with some questions. It is observed in [1] that if $L_{k}(\lambda)$ is perfect and has minimal vectors of ranks 0 or $n$ only, then $k=4$ and the sequence of $L_{k}(\lambda)$ is either $[0,1]$ of $[1,0]$. Are, however, the non-trivial $L_{k}(\lambda)$ all of whose minimal vectors have ranks 0 or $n$ indecomposable? We have already pointed out that there is no overlap (in the sense of isometry) among the lattices sketched in Figure 2. Is it true that all the overlap among the Barnes-Wall lattices is accounted for by Proposition 3.4? Barnes and Wall seem to think so (see [1, page 57]). Finally, and most importantly, are there choices of $G$ (other than elementary 2-groups), $\lambda$ and $\mathbf{C}$ which lead to interesting lattices? For example, are there interesting lattices between $L^{\prime}(G, \lambda)$ and $L^{\prime}(G, \lambda)^{\#}$, where $L^{\prime}(G, \lambda)$ is the lattice of rank $p^{2}-1$ spanned 
by the vectors $\left\{p \mathbf{v}_{C_{1}}-\mathbf{v}_{G}, \ldots, p \mathbf{v}_{C_{k-1}}-\mathbf{v}_{G}\right\}$ of Example 3? Refer to the "gluing" procedure in Section 3 of Chapter 4 in [4].

\section{References}

[1] E. S. Barnes and G. E. Wall, 'Some extreme forms defined in terms of Abelian groups', J. Austral. Math. Soc. 1 (1959), 47-63.

[2] M. Broué and M. Enguehard, 'Une famille infinie de formes quadratiques entieres; leurs groupes d'automorphismes', Ann. Sci. Ecole Norm. Sup. (4) 6 (1973), 17-53.

[3] A. G. Chernyakov, 'Examples of a 32-dimensional even unimodular lattice', J. Soviet Math. 17 (1981), 2068-2075.

[4] J. H. Conway and N. J. A. Sloane, Sphere Packings, Lattices and Groups, Grundlehren der Math. Wissenschaften 290, (Springer-Verlag, Berlin, Heidelberg, New York, 1988).

[5] G. D. Forney, Jr., 'Coset codes. Part I: Introduction and geometrical classification', IEEE Trans. Information Theory 34 (1988), 1123-1151.

[6] G. D. Forney, Jr., 'Coset codes. Part II: Binary lattices and related codes', IEEE Trans. Information Theory 34 (1988), 1152-1187.

[7] H. Koch, 'Unimodular lattices and self-dual codes', Proc. Internat. Congr. Math., (Berkeley, Calif., 1986, Vol. 1, Amer. Math. Soc., Providence, R. I., 1987).

[8] J. Milnor and D. Husemoller, Symmetric Bilinear Forms, (Springer, Berlin, Heidelberg, New York, 1973).

[9] H.-V. Niemeier, 'Definite quadratische Formen der Dimension 24 und Diskriminante 1', J. Number Theory 5 (1973), 142-178.

[10] O. T. O'Meara, Introduction to Quadratic Forms, Grundlehren der Math. Wissenschaften 117, 2nd ed., (Springer, Berlin, Heidelberg, New York, 1971).

[11] O. T. O'Meara, 'On indecomposable quadratic forms', J. Reine Angew. Math. 317 (1980), 120-156.

[12] G. Steinhausen, 'Definite, gerade Bilinearformen der Diskriminante 1', Bonner Mathematische Schriften 76 (Bonn, 1974).

[13] G. E. Wall, 'On the Clifford collineations, transform and similarity groups (IV), an application to quadratic forms', Nagoya Math. 21-22 (1962-1963), 199-222.

\section{University of Notre Dame \\ Notre Dame, Indiana 46556 \\ U.S.A.}

